\title{
SEMICONTINUOUS AND IRRESOLUTE IMAGES OF $S$-CLOSED SPACES
}

\author{
TRAVIS THOMPSON
}

\begin{abstract}
A topological space is $S$-closed if and only if every semi-open cover of $X$ has a finite subcollection whose closures cover $X$. The images of $S$-closed spaces under various mappings are investigated culminating in this main result: A Hausdorff space $X$ is $S$-closed if and only if the irresolute image of $X$ in any Hausdorff space is closed.
\end{abstract}

1. Introduction. In [8] the concept of an $S$-closed space was defined. In this paper, a characterization of Hausdorff $S$-closed spaces are given using a certain class of functions. No separation axioms are assumed unless otherwise specified.

2. Preliminaries. In order for this note to be as self-contained as possible, the following basic definitions are given. A subset $V$ of a topological space is semi-open if and only if $V^{\circ} \subset V \subset \overline{V^{\circ}}$. A topological space $X$ is $S$-closed if and only if every semi-open cover of $X$ has a finite subcollection whose closures cover $X$. A subset $F$ is semiclosed if its complement is semi-open. A function $f: X \rightarrow Y$ is said to be irresolute (semicontinuous) if the inverse image of every semi-open (open) set is semi-open. A topological space is extremally disconnected if the closure of every open set is open. If $A$ is any subset of a topological space $X$, then the semiclosure of $A$, denoted $A$, is the intersection of all semi-closed sets in $X$ that contain $A$. The usual closure of a set $A$ will be denoted by $\bar{A}$ and its interior by $A^{\circ}$.

\section{Main results.}

THEOREM 3.1. A function $f: X \rightarrow Y$ is semicontinuous if and only if for every subset $A$ of $f(\underline{A}) \subset \overline{f(A)}[1$, Theorem 1.16].

THEOREM 3.2. The semicontinuous surjection of an S-closed space onto any Hausdorff space is $\boldsymbol{H}$-closed.

Proof. Let $f: X \rightarrow Y$ be a semicontinuous surjection and $\left\{V_{a}\right\}$ an aribtrary open cover of $Y$. Then $\left\{f^{-1}\left(V_{a}\right)\right\}$ is a semi-open cover of $X$. By hypothesis, there exists a finite subfamily such that $\cup_{1}^{n} \overline{f^{-1}\left(V_{a_{i}}\right)}=X$. Notice that $\cup_{1}^{n} f^{-1}\left(V_{a_{i}}\right)$ being dense in $X$ implies $\cup_{1}^{n} f^{-1}\left(V_{a_{i}}\right)=X$. By Theorem 3.1,

Received by the editors July 26, 1976.

AMS (MOS) subject classifications (1970). Primary 54D20, 54D30; Secondary 54G05.

Key words and phrases. $S$-closed, extremally disconnected, semicontinuous, irresolute. 


$$
\begin{aligned}
& Y=f(X)=f\left[\bigcup_{1}^{n} f^{-1}\left(V_{a_{i}}\right)\right] \subset \overline{\left[\bigcup_{1}^{n} f^{-1}\left(V_{a_{i}}\right)\right]} \\
& =\overline{\bigcup_{1}^{n} V_{a_{i}}}=\bigcup_{1}^{n} \overline{V_{a_{i}}} \text {. }
\end{aligned}
$$

Therefore, $Y$ is $H$-closed.

The proof of Theorem 3.2 remains valid without the assumption of $Y$ being Hausdorff; that is, $Y$ enjoys the " $H$-closed covering property."

Corollary 3.3. The semicontinuous surjection of an $S$-closed space onto any regular space is compact.

THEOREM 3.4. A function $f: X \rightarrow Y$ is irresolute if and only if for every subset $A$ of $X, f(\underline{A}) \subset f(A)[3$, Theorem 1.5].

THEOREM 3.5. If $f: X \rightarrow Y$ is an irresolute surjection from an $S$-closed space $X$, then $Y$ is $S$-closed.

Proof. Let $\left\{V_{a}\right\}$ be a semi-open cover of $Y$. Then $\left\{f^{-1}\left(V_{a}\right)\right\}$ is a semiopen cover of $X$ and, by hypothesis, has a finite subfamily such

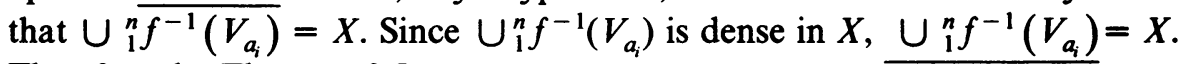
Therefore, by Theorem 3.5,

$$
\begin{aligned}
Y & =f(X)=f \frac{\left[\bigcup_{1}^{n} f^{-1}\left(V_{a_{i}}\right)\right]}{n} \subset f\left[\bigcup_{1}^{n} f^{-1}\left(V_{a_{i}}\right)\right] \\
& =\bigcup_{1}^{n} V_{a_{i}} \subset \bigcup_{1}^{n} \overline{V_{a_{i}}} .
\end{aligned}
$$

Hence, $Y$ is $S$-closed.

COROLlARY 3.6. $S$-closed is a semitopological property, and hence a topological property [3, Theorem 1.15].

We note that the $S$-closed property is not, in general, preserved by continuous functions: $\beta R$ is the continuous image of $\beta N$, but $\beta N$ is $S$-closed $[8$, Theorem 5] and $\beta R$ is not.

The following lemma is well known and will be stated without proof.

LEMMA 3.7. A topological space $X$ is extremally disconnected if and only if every two disjoint open sets in $X$ have disjoint closures.

Definition 3.8. A filterbase $F$ is said to $s$-accumulate to a point $x$ if for every semi-open set $V$ containing $x$ and for every $F_{a} \in F, F_{a} \cap \bar{V} \neq \varnothing$.

In the following theorem we use the fact that an $S$-closed Hausdorff space is extremally disconnected [8, Theorem 7$]$.

THEOREM 3.9. A space is $S$-closed if and only if every filterbase has an $s$-accumulation point [8, Theorem 2]. 
THEOREM 3.10. The irresolute image of any $S$-closed Hausdorff space in any Hausdorff space is closed.

Proof. Let $f: X \rightarrow Y$ be an irresolute function from an $S$-closed space $X$ to a Hausdorff space $Y$. Let $y \in \overline{f(X)}$ and $N(y)$ be the open neighborhood filterbase about $y$. By hypothesis, the filterbase $F=f^{-1}[N(y)]$ has an $s-$ accumulation point $x$. We claim that the filterbase $f(F)$ accumulates to $f(x)$ in the usual sense. Indeed, let $V$ be any open set containing $f(x)$. Then $f^{-1}(V)$ is a semi-open set containing $x$, and therefore for every $W \in$ $N(y), f^{-1}(W) \in F$, and $f^{-1}(W) \cap \overline{f^{-1}(V)} \neq \varnothing$. But by Lemma 3.7, we have $f^{-1}(W)^{\circ} \cap f^{-1}(V)^{\circ} \neq \varnothing$. Therefore,

$$
\varnothing \neq f\left[f^{-1}(W)^{0} \cap f^{-1}(V)^{0}\right] \subset f\left[f^{-1}(W) \cap f^{-1}(V)\right] \subset W \cap V .
$$

Since $W$ and $V$ were arbitrarily chosen, we have that $f(F)$ accumulates to $f(x)$ in the usual sense. But $f(F)$ is a finer filterbase than $N(y)$, hence $N(y)$ accumulates to $f(x)$. Since $N(y)$ obviously converges to $y$, we have by the Hausdorff property that $f(x)=y$. Hence, $y \in f(X)$ and $f(X)$ is closed in $Y$.

THEOREM 3.11. If every irresolute image of a Hausdorff space $X$ in any Hausdorff space $Y$ is closed, then $X$ is $S$-closed.

Proof. Suppose that $X$ is not $S$-closed. Then by Theorem 3.9 there exists a filterbase $F$ with no $s$-accumulation point. This implies that for every $x \in X$, there exist an open set $V(x)$ about $x$ and an element $F_{a(x)}$ of $F$ such that $F_{a(x)} \cap \overline{V(x)}=\varnothing$. Let $S=$ all finite intersections of sets of the form $(X-\overline{V(x)})\}$. It is evident that $S$ forms an open filterbase. Select an object $\infty$ not in $X$ and consider the space $\hat{X}=X \cup\{\infty\}$ with the following topology: neighborhoods of points in $X$ are unchanged, and a basic neighborhood system of $\infty$ is $N(\infty)=\left\{S_{b} \cup\{\infty\} \mid S_{b} \in S\right\}$. It is easy to verify that $\hat{X}$ is Hausdorff. Considering the inclusion map $i: X \rightarrow \hat{X}$, we see that $i$ is irresolute and that $i(X)$ is not closed in $\hat{X}$. Therefore, the theorem follows by contraposition.

Combining the results of Theorems 3.10 and 3.11 we have the following characterization.

Corollary 3.12. A Hausdorff space $X$ is $S$-closed if and only if the irresolute image of $X$ in any Hausdorff space is closed.

\section{REFERENCES}

1. S. Gene Crossley and S. K. Hildebrand, Semi-closed sets and semi-continuity in topological spaces, Texas J. Sci. 22 (1971), 123-126.

2. __ Semi-closure, Texas J. Sci. 22 (1971), 99-112.

3. __ Semi-topological properties, Fund. Math. 74 (1972), 233-254.

4. J. Dugundji, Topology, Allyn and Bacon, Boston, 1966.

5. T. R. Hamlett, Semi-continuous and irresolute functions, Texas Academy of Science, Vol. 27, Nos. 1, 2. 
6. Y. Isomichi, New concepts in the theory of topological space-supercondensed set, subcondensed set, and condensed set, Pacific J. Math. 38(1971), 657-668.

7. Norman Levine, Semi-open sets and semi-continuity in topological spaces, Amer. Math. Monthly 70 (1963), 36-41.

8. Travis Thompson, S-closed spaces, Proc. Amer. Math. Soc. CO (1970), 335-338.

9. S. Willard, General topology, Addison-Wesley, Reading, Mass., 1970. MR 41 \#9173.

Departmint of Mathematics, University of Arkansas, Fayetteville, Arkansas 72701 